УДК 622.279 .72

\title{
ИНЖЕКЦИЯ ЖИДКОГО ДИОКСИДА УГЛЕРОДА В ПЛАСТ, НАСЫЩЕННЫЙ МЕТАНОМ И ЕГО ГАЗОГИДРАТОМ
}

\author{
Хасанов Марат Камилович', \\ hasanovmk@mail.ru \\ Столповский Максим Владимирович2,1, \\ s_maxim.pmm@mail.ru \\ Гималтдинов Ильяс Кадирович², \\ iljas_g@mail.ru \\ 1 Стерлитамакский филиал Башкирского государственного университета, \\ Россия, 453103, г. Стерлитамак, пр. Ленина, 49. \\ 2 Уфимский государственный нефтяной технический университет, \\ Россия, 450062, г. Уфа, ул. Космонавтов, 1.
}

\begin{abstract}
Актуальность исследования связана с разработкой теоретических основ технологий добычи газа из газогидратных месторождений методом замещения. Рассматривается метод инжекции диоксида углерода, позволяющий значительно снизить энергетические затраты на разработку природных газогидратных залежей.

Целью исследования является выяснение особенностей протекания процесса замещения метана диоксидом углерода в газогидрате при инжекции жидкого диоксида углерода в газогидратный пласт

Объект: пористый пласт конечной протяженности, насыщенный метаном и его газогидратом, исходные давление и температура которого соответствуют условиям стабильного существования газогидрата метана.

Методы. На основе уравнений механики сплошной среды построена математическая модель тепломассопереноса в природном пласте, сопровождающаяся замещением метана на диоксид углерода в газогидрате. Принято, что в рассматриваемом случае в пласте возникают две характерные зоны, разделенные подвижной границей фазовых переходов. В первой (ближней) зоне поры насыщены жидкой двуокисью углерода и ее газогидратом, а во второй (дальней) зоне содержатся метан и его газогидрат. Результаты. Получены численные решения для полей давления и температуры при инжекции жидкого диоксида углерода в газогидратный пласт конечной протяженности. Построены зависимости температуры на границе замещения от давления инжекции и проницаемости пласта. Установлено, что при достаточно низких значениях давления инжекции и проницаемости величина температуры на границе замещения может подниматься выше равновесного значения температуры диссоциации газогидрата метана на газ и воду. Это соответствует возникновению второй подвижной межфазной границы, на которой происходит разпожение газогидрата метана. Найдена зависимость предельной температуры закачиваемой жидкой двуокиси углерода, выше которой необходимо учитывать образование смеси метана и воды, от значений давления на правой и левой границах пласта и его проницаемости. Установлено, что режим с разложением газогидрата реализуется при высоких значениях давления на правой границе пласта и низких значениях проницаемости и давления, под которым закачивается двуокись углерода. Получены зависимости скорости границы замещения, а также времени полной замены газогидрата метана на газогидрат диоксида углерода во всем пласте от давления на правой и левой границах пласта, а также от его проницаемости.
\end{abstract}

Ключевые слова:

Газогидрат метана, диоксид углерода, фильтрация, пористый пласт, замещение.

\section{Введение}

В настоящее время природные газовые гидраты рассматриваются как один из возможных и практически неисчерпаемых источников газа (метана). Использование традиционных методов добычи газа из газогидратных залежей (снижение давления на забое добывающей скважины, нагрев пласта или закачка ингибиторов) зачастую бывает экономически нецелесообразным, а также может оказать неблагоприятное воздействие на окружающую среду. В связи с этим был предложен новый метод добычи природного газа, заключающийся в инжекции углекислого газа (жидкого или газообразного) в метаногидратные пласты. Данный метод обусловлен тем, что гидрат $\mathrm{CO}_{2}$ термодинамически более стабилен, чем гидрат $\mathrm{CH}_{4}$ [1-11]. Поэтому диоксид углерода может замещать метан в его гидрате. При этом наряду с извлечением при- родного газа может частично решаться проблема утилизации диоксида углерода, вносящего значительный вклад в развитие парникового эффекта.

Результаты экспериментальных исследований образования газогидрата $\mathrm{CO}_{2}$ и замещения метана в газогидрате на диоксид углерода приведены, в частности, в работах [1-14]. В работах [15-17] представлены результаты математического моделирования процесса инжекции диоксида углерода в полубесконечный пористый пласт. Для таких пластов влияние правой границы на процессы тепломассопереноса оказывается несущественным. Однако для более детального описания процессов, происходящих в пористых средах при инжекции жидкого диоксида углерода, необходимо учитывать конечную протяженность гидратонасыщенных пластов. В настоящей работе представлены результаты численного моделирования инжекции 
жидкой двуокиси углерода в пористый пласт конечной протяженности, изначально насыщенный метаном и его гидратом.

\section{Постановка задачи}

Рассмотрим пористый пласт длины $L(0 \leq x \leq L)$, кровля и подошва которого непроницаемы и теплоизолированы. Пусть пласт в начальный момент времени насыщен метаном и его гидратом, температура $T_{0}$ и давление $p_{0}$ которых соответствуют условиям существования метаногидрата. Через его левую границу $(x=0)$ закачивается жидкий диоксид углерода, давление $p_{e}$ и температура $T_{e}$ которого соответствуют условиям стабильного существования гетерогенной смеси жидкой двуокиси углерода и ее гидрата.

Условия стабильного существования газовых гидратов принято изображать с помощью фазовых диаграмм [18]. На рис. 1 представлена диаграмма фазового равновесия гидратов метана и диоксида углерода.

\section{$p$, МПа}

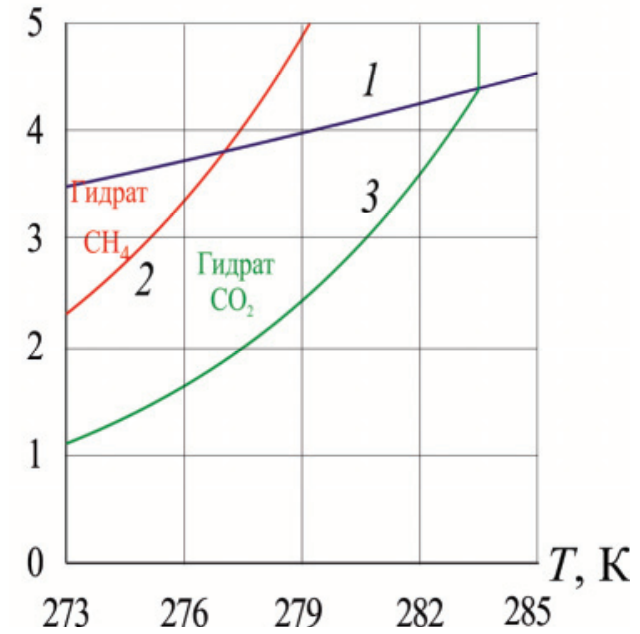

Pис.1. Диаграмла фазового равновесия гидратов метана и диоксида углерода

Fig. 1. Diagram of phase equilibrium of methane hydrates and carbon dioxide

На рис. 1 кривые 2 и 3 соответствуют условиям фазового равновесия систем « $\mathrm{CH}_{4}$ (газ) - гидрат $\mathrm{CH}_{4}$ - вода» и « $\mathrm{CO}_{2}$ (газ) - гидрат $\mathrm{CO}_{2}$ - вода». Линия 1 определяет равновесное состояние между жидким и газообразным диоксидом углерода. Таким образом, начальным параметрам системы $\left(T_{0}, p_{0}\right)$ на фазовой диаграмме соответствует точка, лежащая выше кривой 2, а параметрам инжектируемой жидкости $\left(T_{e}, p_{e}\right)$ - точка, лежащая выше линии 1.

В результате нагнетания через левую границу пласта жидкого диоксида углерода в пласте образуются две характерные области (рис. 2): ближняя, содержащая жидкий $\mathrm{CO}_{2}$ и его гидрат, и дальняя, содержащая $\mathrm{CH}_{4}$ и его гидрат. При этом возникает устойчивая подвижная граница фазового перехода $x_{(n)}$, на которой происходит замещение метана в газогидрате двуокисью углерода. Устойчивость границы фазового перехода обусловлена ламинарностью течения в пористой среде, а также тем, что вязкость жидкой двуокиси углерода на порядок выше вязкости метана. В рассматриваемой задаче будем пренебрегать диффузией двуокиси углерода и метана, т. к. вследствие непрерывной закачки диоксида углерода в пласт интенсивность массопереноса, обусловленного фильтрацией в проницаемой пористой среде, значительно превышает интенсивность массопереноса, обусловленного диффузией.

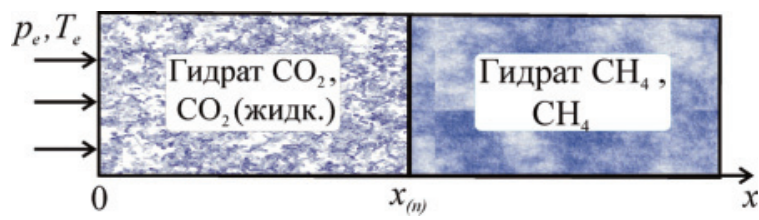

Puс. 2. Схема областей, форлируемых при закачке жидкой двуокиси углерода

Fig. 2. Areas formed at injection of liquid carbon dioxide

\section{Основные уравнения}

При описании процессов тепломассопереноса примем следующие допущения: пористость постоянна; газогидраты метана и диоксида углерода неподвижны и несжимаемы; температура пористой среды и насыщающего ее вещества постоянна. Гидраты $\mathrm{CO}_{2}$ и $\mathrm{CH}_{4}$ являются двухкомпонентными системами с массовой концентрацией газа $G_{c}$ и $G_{m}$ соответственно. При этом газ (метан) считается калорически совершенным, а жидкость (двуокись углерода) - упругой жидкостью.

Система основных уравнений, представляющая собой уравнения сохранения массы метана и двуокиси углерода, энергии, закон Дарси, уравнение состояния калорически совершенного газа и упругой жидкости в плоскоодномерном приближении, при отмеченных допущениях имеет вид $[15-17,19-24]$ :

$$
\begin{gathered}
\frac{\partial}{\partial t}\left(\rho_{j} \phi S_{j}\right)+\frac{\partial}{\partial x}\left(\rho_{j} \phi S_{j} v_{j}\right)=0, \\
\rho C \frac{\partial T}{\partial t}+\rho_{j} \tilde{N}_{j} \phi S_{j} v_{j} \frac{\partial T}{\partial x}-\frac{\partial}{\partial x}\left(\lambda \frac{\partial T}{\partial x}\right)=0, \\
\phi S_{j} v_{j}=-\frac{k_{j}}{\mu_{j}} \frac{\partial p}{\partial x}, \\
\rho_{c}=\rho_{0 c}\left(1+\beta\left(p-p_{0}\right)\right), \rho_{m}=p / R_{m} T, k_{j}=k_{0} S_{j}^{3} .
\end{gathered}
$$

Здесь $\phi$ - пористость; $p$ - давление; $T$ - температура; $\rho_{j}, S_{j}, v_{j}, k_{j}, C_{j}$ и $\mu_{j}$ - соответственно плотность, насыщенность, скорость, фазовая проницаемость, удельная теплоемкость и динамическая вязкость метана $(j=m)$ и диоксида углерода $(j=c) ; R_{m}$ - газовая постоянная метана; $\rho_{0 c}-$ плотность жидкого диоксида углерода, соответствующая давлению $p_{0}$; $\beta$ - коэффициент объемного сжатия $\mathrm{CO}_{2} ; k_{0}$-абсолютная проницаемость; $\rho C$ и $\lambda-$ удельная объе- 
мная теплоемкость и коэффициент теплопроводности системы.

Условия баланса массы диоксида углерода и метана на границе вытеснения метана диоксидом углерода с учетом замещения $\mathrm{CH}_{4}$ на $\mathrm{CO}_{2}$ в составе газогидрата имеют вид $[15,16]$ :

$$
\begin{gathered}
\phi \rho_{c} S_{c}\left(v_{c}-\dot{x}_{(n)}\right)=\phi \rho_{h c} S_{h c} G_{c} \dot{x}_{(n)}, \\
\phi \rho_{m} S_{m}\left(v_{m}-\dot{x}_{(n)}\right)=\phi \rho_{h m} S_{h m} G_{m} \dot{x}_{(n)},
\end{gathered}
$$

где $\rho_{h j}, S_{h j}$ - плотность и насыщенность пор для газогидрата диоксида углерода $(j=c)$ и газогидрата метана $(j=m), \dot{x}_{(n)}-$ скорость движения границы, на которой происходит замещение. Нижний индекс $n$ относится к параметрам на границе замещения.

С учетом последней системы уравнений и закона Дарси условия баланса массы и тепла на границе между первой и второй областями можно записать следующим образом:

$$
\begin{gathered}
-\frac{k_{c}}{\mu_{c}} \frac{\partial p_{(1)}}{\partial x}=\phi\left(\frac{\rho_{h c}}{\rho_{c}} S_{h c} G_{c}+S_{c}\right) \dot{x}_{(n)}, \\
-\frac{k_{m}}{\mu_{m}} \frac{\partial p_{(2)}}{\partial x}=\phi\left(\frac{\rho_{h m}}{\rho_{m}} S_{h m} G_{m}+S_{m}\right) \dot{x}_{(n)}, \\
\phi S_{h c} \rho_{h c}\left(1-G_{c}\right) \dot{x}_{(n)}=\phi S_{h m} \rho_{h m}\left(1-G_{m}\right) \dot{x}_{(n)}, \\
\lambda \frac{\partial T_{(1)}}{\partial x}-\lambda \frac{\partial T_{(2)}}{\partial x}=\phi\left(\rho_{h c} L_{h c} S_{h c}-\rho_{h c} L_{h m} S_{h m}\right) \dot{x}_{(n)} .
\end{gathered}
$$

Здесь $L_{h j}$ - теплота образования газогидратов диоксида углерода $(j=c)$ и метана $(j=m), p_{(i)}$ и $T_{(i)}-$ давление и температура в первой $(i=1)$ и второй $(i=2)$ областях.

Начальные условия, а также условия на границах пласта имеют вид:

$$
\begin{gathered}
t=0: p=p_{0}, \quad T=T_{0} \quad(0 \leq x \leq L) ; \\
x=0: p=p_{e}, T=T_{e} \quad(t>0) ; \\
x=L: p=p_{0}, \frac{\partial T}{\partial x}=0 \quad(t>0) .
\end{gathered}
$$

Пусть исходная объемная насыщенность пор гидратом метана равна $S_{h m}=v$, тогда из второго уравнения системы (2) для объемной насыщенности гидратом $\mathrm{CO}_{2}$ в первой области получим:

$$
S_{h c}=\rho_{h m}\left(1-G_{m}\right)\left(\rho_{h c}\left(1-G_{c}\right)\right)^{-1} v .
$$

На основе системы (1) уравнения пьезопроводности и температуропроводности для каждой из областей запишутся в виде:

$$
\begin{gathered}
\frac{\partial p_{(1)}}{\partial t}=\frac{k_{c}}{\mu_{c} \phi \beta S_{c} \exp \left(\beta\left(p_{1}-p_{0}\right)\right)} \times \\
\times \frac{\partial}{\partial x}\left(\exp \left(\beta\left(p_{1}-p_{0}\right)\right) \frac{\partial p_{(1)}}{\partial x}\right), \\
\frac{\partial T_{(1)}}{\partial t}=\chi^{(T)} \frac{\partial}{\partial x}\left(\frac{\partial T_{(1)}}{\partial x}\right)+ \\
+\frac{\rho_{0 c} \exp \left(\beta\left(p_{1}-p_{0}\right)\right) C_{c} k_{c}}{\rho \tilde{N} \mu_{c}} \frac{\partial p_{(1)}}{\partial x} \frac{\partial T_{(1)}}{\partial x},
\end{gathered}
$$

$$
\begin{gathered}
\frac{\partial p_{2}}{\partial t}=\frac{k_{m}}{\phi S_{m} \mu_{m}} \frac{\partial}{\partial x}\left(p_{2} \frac{\partial p_{2}}{\partial x}\right), \\
\frac{\partial T_{(2)}}{\partial t}=\chi^{(T)} \frac{\partial}{\partial x}\left(\frac{\partial T_{(2)}}{\partial x}\right)+\frac{p_{(2)} \tilde{N}_{m} k_{m}}{R_{m} T_{(2)} \rho C \mu_{m}} \frac{\partial p_{(2)}}{\partial x} \frac{\partial T_{(2)}}{\partial x},
\end{gathered}
$$

где $\chi^{(T)}=\lambda / \rho C-$ коэффициент температуропроводности пласта; индексы $i=1,2$ относятся к параметрам первой и второй зон соответственно.

Полученная система уравнений (4), (5) с начальными и граничными условиями (3), а также условиями (2) на подвижной границе фазовых переходов является замкнутой. Для ее решения каждое из дифференциальных уравнений представляется в виде неявной конечно-разностной схемы. При этом используем метод ловли фронта в узел пространственной сетки, суть которого заключается в том, что за неизвестный и определяемый в ходе решения задачи временной шаг фронт фазового перехода перемещается по координатной сетке ровно на один шаг. Решение системы уравнений проводилось на каждом временном слое с помощью метода прогонки в сочетании с методом итераций до достижения заданной точности, как по давлению, так и по температуре. Число точек разбиения по пространственной координате полагалось равным $n=2000$.

\section{Результаты расчетов}

На рис. 3 для момента времени $t=30$ сут. представлены распределения температуры и давления в пласте при нагнетании жидкой двуокиси углерода под давлением $p_{e}=4,0 \mathrm{MПа.} \mathrm{Для} \mathrm{других} \mathrm{параметров}$ приняты значения [15-24]: $\phi=0,2, v=0,2, k_{0}=10^{-16} \mathrm{M}^{2}$, $p_{0}=3,5 \mathrm{M \Pi а}, T_{0}=274 \mathrm{~K}, \quad \rho C=2,5 \cdot 10^{6}$ Дж $/\left(\mathrm{K} \cdot \mathrm{M}^{3}\right)$, $\lambda=2$ Вт $/(\mathrm{m} \cdot \mathrm{K}), G_{c}=0,28, G_{m}=0,13, R_{m}=520$ Дж $/(\kappa \Gamma \cdot К)$, $\rho_{h c}=1100 \kappa \Gamma / \mathrm{M}^{3}, \quad \rho_{h m}=900 \kappa \Gamma / \mathrm{M}^{3}, \quad \rho_{c 0}=890 \mathrm{\kappa} / \mathrm{M}^{3}$, $\beta=10^{-8} \Pi \mathrm{a}^{-1}, \mu_{c}=10^{-4} \Pi \mathrm{a} \cdot \mathrm{c}, \mu_{m}=10^{-5} \Pi \mathrm{a} \cdot \mathrm{c}$, $L_{h c}=3,54 \cdot 10^{5}$ Дж/к г, $L_{h m}=4,5 \cdot 10^{5}$ Дж/к г, $C_{c}=2600$ Дж $/(\kappa \Gamma \cdot \mathrm{K}), C_{m}=1560$ Дж/(кг.К), $L=100 \mathrm{м}$.

Как следует из рис. $3, a$, при относительно невысокой температуре закачиваемой двуокиси углерода температура в области, содержащей метан и его гидрат, лежит ниже его равновесной температуры разложения (штриховая линия), определяемой выражением [25]:

$$
\ln p=A-B / T \text {, }
$$

где $A=29,112, B=7694,30$. Следовательно, в этом случае решение с одной границей фазового перехода является термодинамически непротиворечивым. При увеличении температуры закачиваемого диоксида углерода (рис. $3, b$ ) величина температуры в области, содержащей газогидрат метана, на некотором участке поднимается выше равновесного значения температуры его разложения. В этом случае решение с одной подвижной границей фазового перехода (границей замещения) не позволяет построить термодинамически непротиворечивое решение. Поэтому необходимо вводить дополнительную границу фазовых превращений, на которой происходит диссоциация газогидрата $\mathrm{CH}_{4}$ на 
$a$
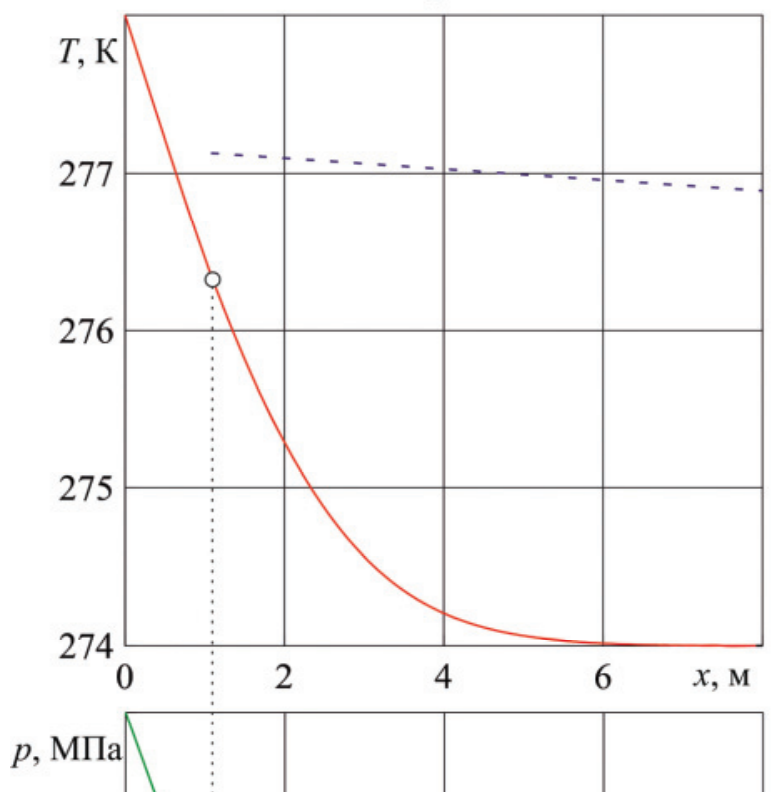

3.9

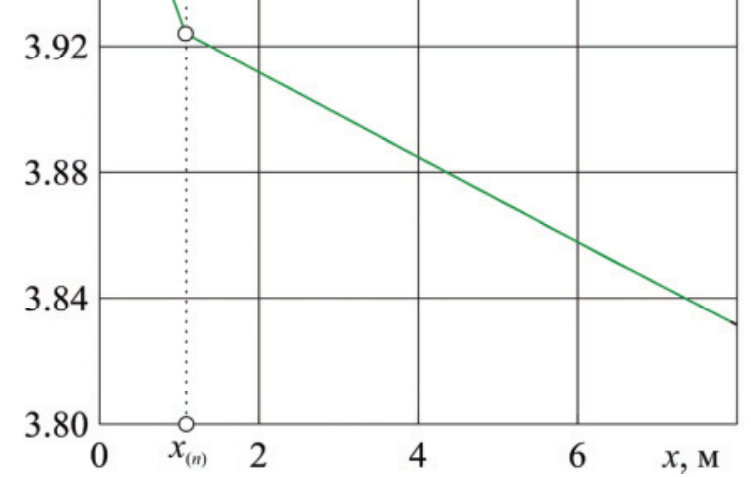

$b$
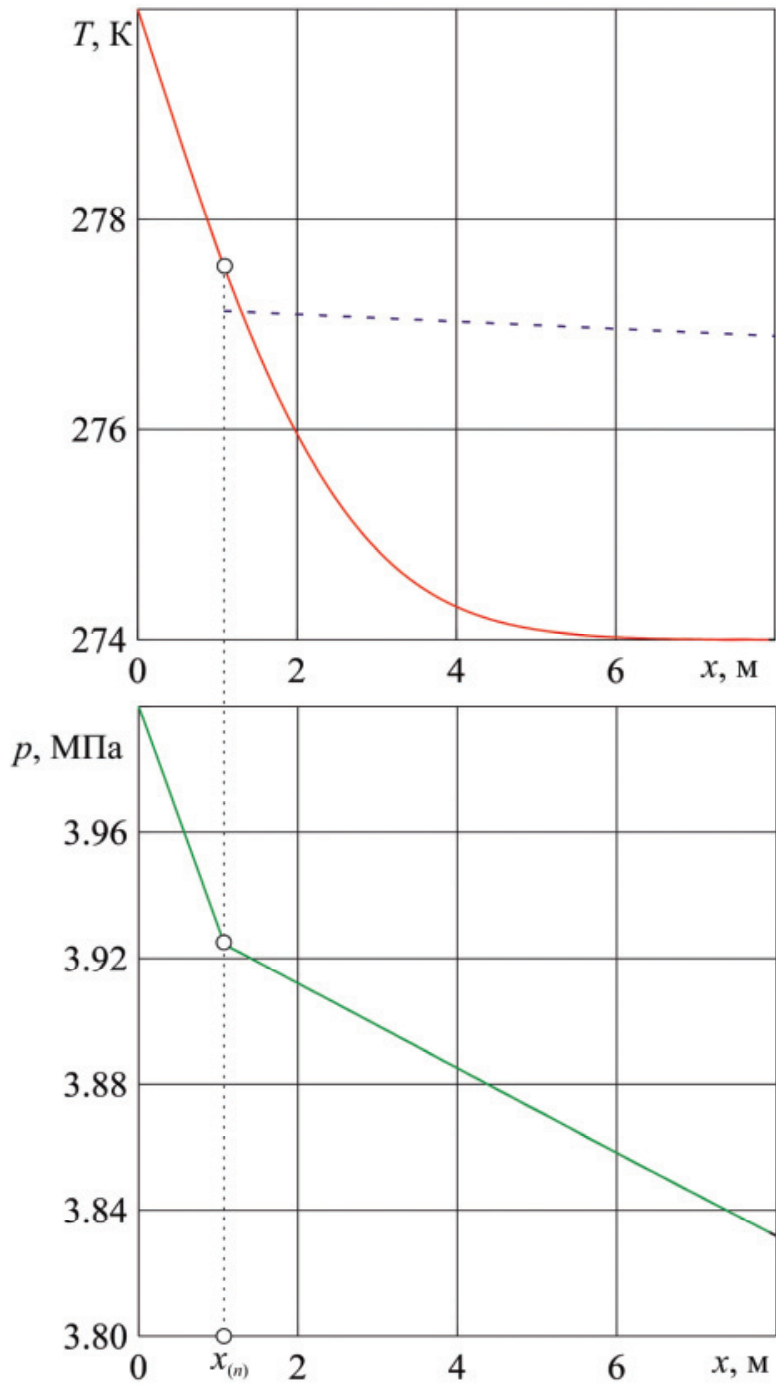

Puс. 3. Распределение телпературы и давления пласта в момент времени $t=30$ сут. $T_{e}=278 \mathrm{~K}(\mathrm{a})$ u $280 \mathrm{~K}(\mathrm{~b})$

Fig. 3. Distribution of the temperature and pressure of the reservoir at $t=30$ days. $T_{e}=278 \mathrm{~K}(\mathrm{a}) \mathrm{u} 280 \mathrm{~K}(\mathrm{~b})$

газ и воду. В этом случае в пласте будет возникать промежуточная область, содержащая метан и воду в свободном состоянии.

На рис. 4 приведена зависимость температуры и координаты границы замещения от давления, под которым закачивается диоксид углерода $(a)$, и проницаемости пласта $(b)$ в момент времени $t=10$ сут. Штриховая линия показывает равновесную температуру разложения газогидрата метана, соответствующую вычисленному значению давления на границе замещения.

В соответствии с рис. 4 при понижении давления инжекции и проницаемости пласта температура на границе замещения возрастает. Это обусловлено снижением скорости границы замещения и соответственно возрастанием влияния более горячей левой границы на величину температуры данной границы. Поэтому при достаточно низких значениях проницаемости пласта и давления, под которым закачивается двуокись углерода, величина температуры пласта на границе замещения может подниматься выше равновесного значения температуры диссоциации газогидрата метана. Это соответствует возникновению дополнительной области, насыщенной продуктами разложения газогидрата метана.

Были проведены расчеты для определения предельного значения температуры закачиваемой двуокиси углерода, выше которой необходимо учитывать образование смеси метана и воды. На рис. 5 представлена зависимость предельного значения температуры инжектируемой двуокиси углерода от давления, под которым закачивается диоксид углерода $(a)$, и проницаемости пласта $(b)$ для момента времени $t=10$ сут. после начала закачки. Кривые 1 и 2 соответствуют значениям давления $p_{0}$ на правой границе пласта 3,2 и 3,5 МПа. Согласно рис. 5 , при повышении давления инжекции и проницаемости предельное значение температуры возрастает, причем тем быстрее, чем ниже 
$a$
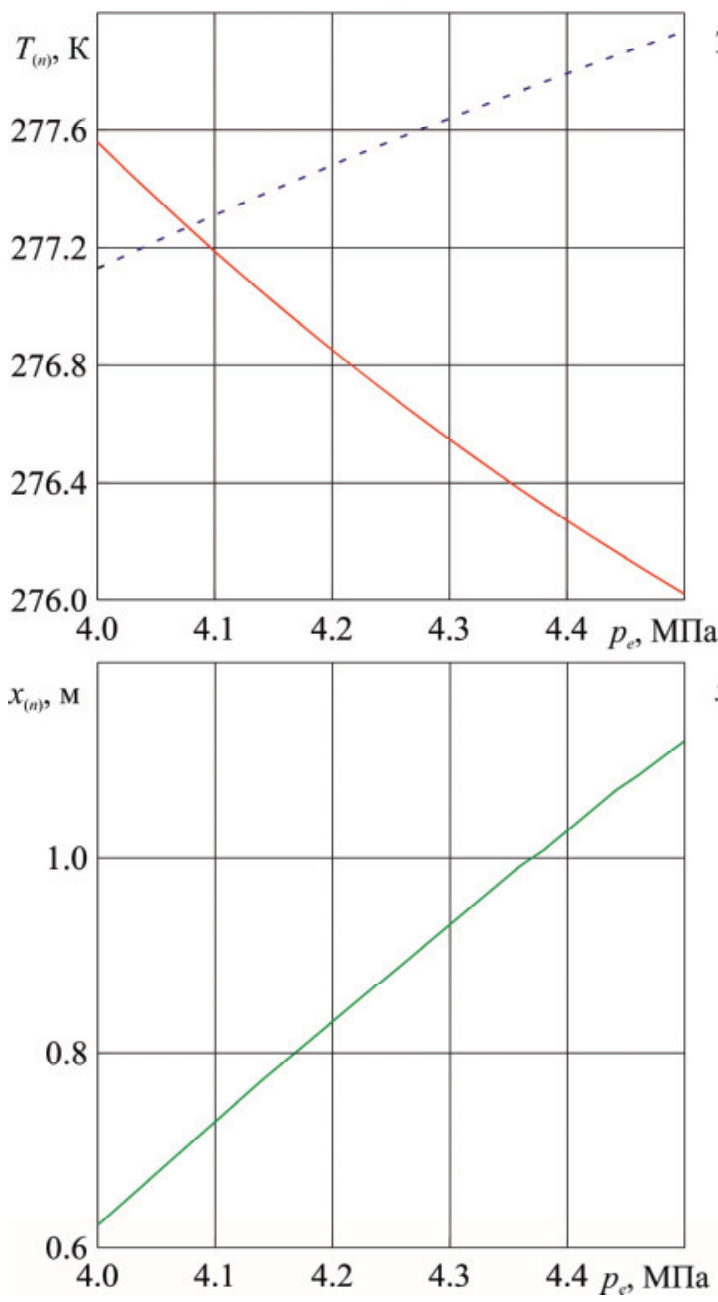

$b$
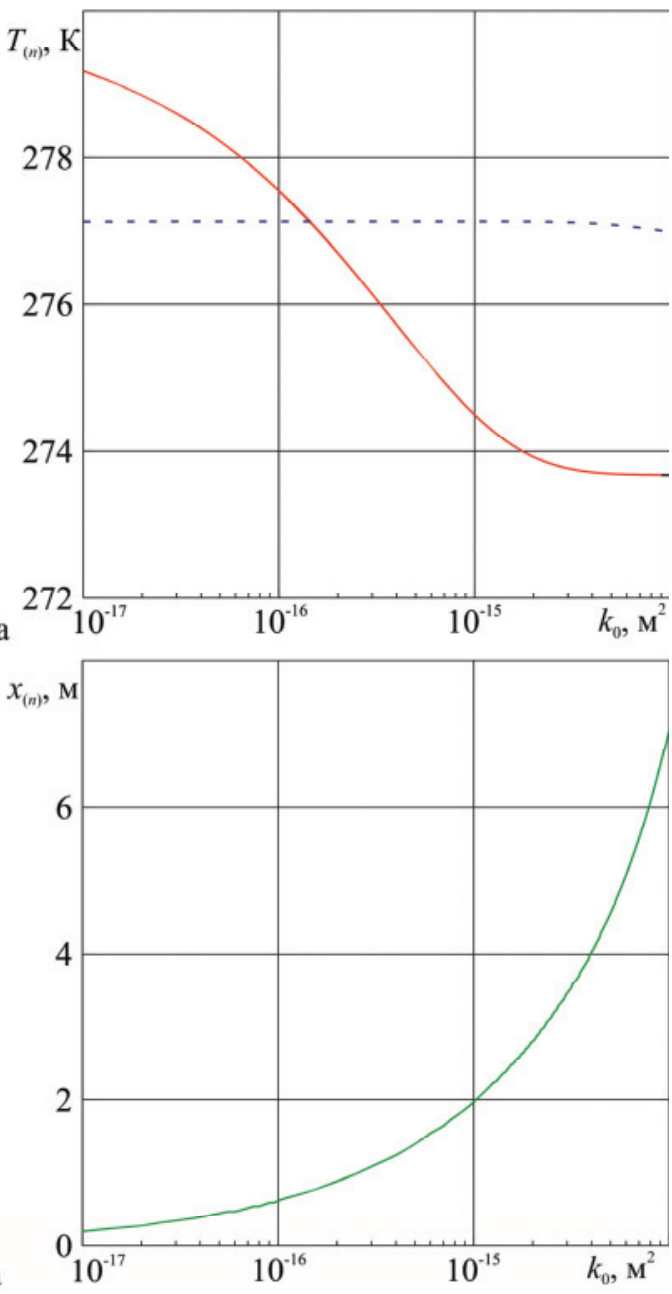

Pис. 4. Зависимость телпературы и координаты границы залещения от давления, под которым закачивается двуокись углерода (а), и проницаемости пласта (b)

Fig. 4. Dependence of temperature and substitution boundary coordinate on the pressure at which carbon dioxide is injected (a), and on formation permeability $(b)$
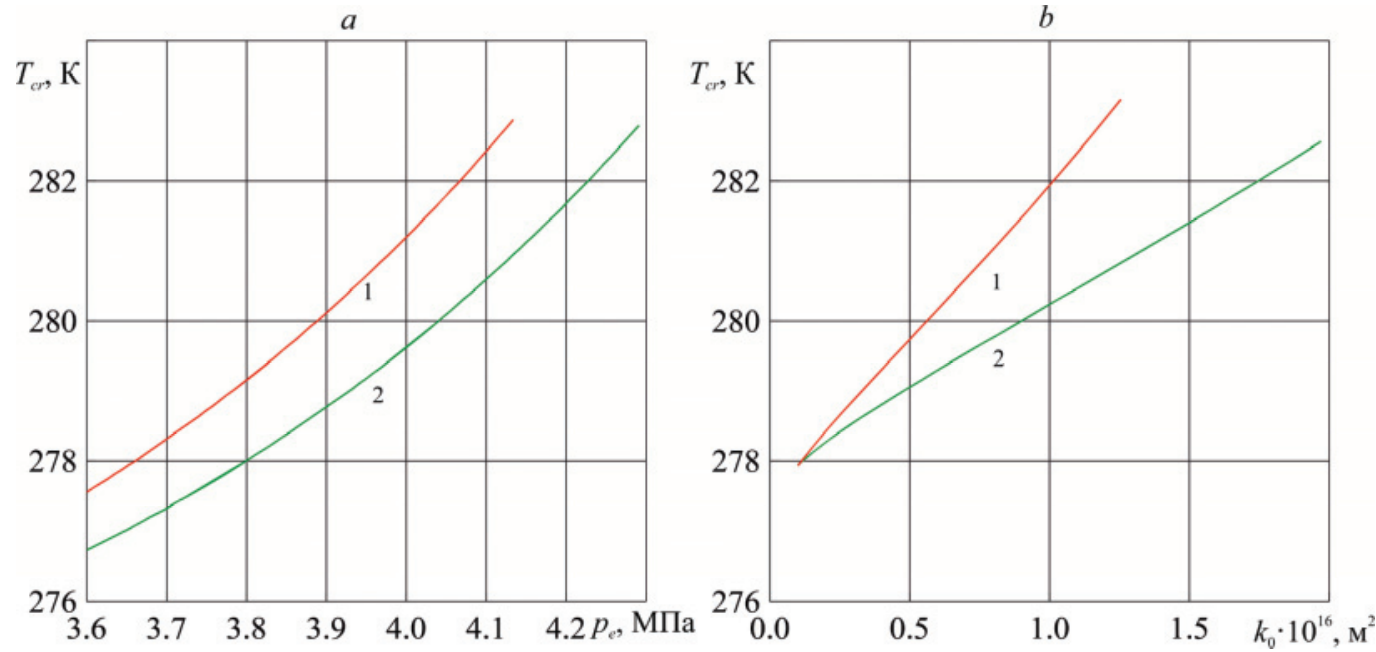

Pис.5. Зависимость предельного значения телпературы от давления инжекции двуокиси углерода (а) и абсолютной проницаелости пласта (b) для момента времени $t=10$ сут. $p_{0}=3,2$ МПа (кривая 1) и 3,5 МПа (кривая 2)

Fig. 5. Dependence of the temperature limit on carbon dioxide injection pressure ( $a$ ) and the absolute permeability of the formation ( $b$ ) for the time instant $t=10$ days: $p_{0}=3,2 \mathrm{MPa}$ (curve 1 ) and 3,5 $\mathrm{MPa}$ (curve 2 ) 
$a$

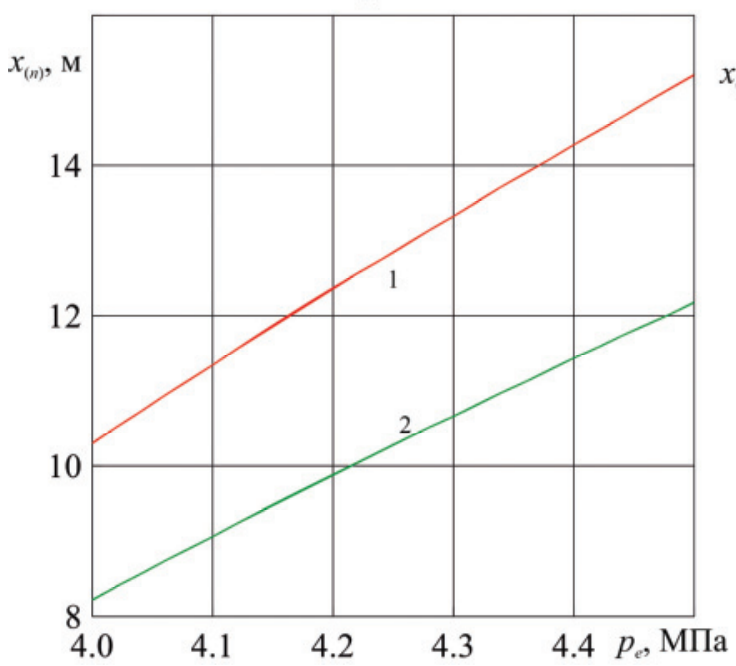

$b$

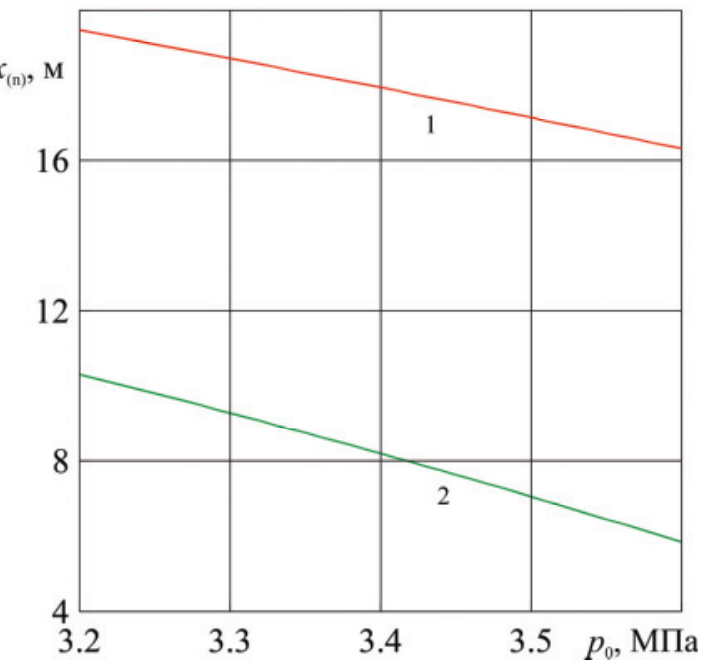

Pис. 6. Зависимость координаты границы фазового перехода от: а) давления инжекиии: $k_{0}=10^{-14} \mathrm{M}^{2}(\kappa р и в а я ~ 1), 7 \cdot 10^{-15} \mathrm{M}^{2}(\kappa р и в а я ~ 2)$; b) давления на правой границе пласта: $p_{e}=4$ МПа (кривая 1), 5 МПа (кривая 2)

Fig. 6. Dependence of the phase transition boundary coordinate on: a) injection pressure: $k_{0}=10^{-14} \mathrm{~m}^{2}($ curve 1$\left.), 7 \cdot 10^{-15} \mathrm{~m}^{2}(\mathrm{curve} 2) ; b\right)$ pressure on the right boundary of the layer: $p_{e}=4 \mathrm{MPa}$ (curve 1), $5 \mathrm{MPa}$ (curve 2 )

давление на правой границе пласта. Следовательно, рассматриваемый в данной работе режим с замещением метана на двуокись углерода в газогидрате характерен для высоких значений перепада давления $\Delta p=p_{e}-p_{0}$ в пласте и высокопроницаемых пористых сред. Это объясняется тем, что в этом случае скорость фильтрации диоксида углерода и соответственно границы замещения в газогидрате метана на двуокись углерода выше скорости распространения температурного фронта от более нагретой левой границы пласта. Поэтому область, насыщенная метаном и его газогидратом, не успевает прогреваться до значений температур, соответствующих разложения газогидрата метана на газ и воду.

На рис. 6 для момента времени $t=10$ сут. представлены зависимости координаты границы фазового перехода от значений давления на левой $(a)$ и правой $(b)$ границах пласта. Температура нагнетаемой двуокиси углерода полагалась равной $T_{e}=276 \mathrm{~K}$. Как следует из рисунка, с ростом давления инжекции и проницаемости, а также с уменьшением давления на правой границе пласта координата границы фазовых переходов увеличивается. Это объясняется тем, что скорость движения границы фазового перехода лимитируется скоростью поступления $\mathrm{CO}_{2}$ к границе фазового перехода, которая тем выше, чем больше перепад давления и выше проницаемость пласта.

Зависимость времени полного перехода гидрата метана в гидрат диоксида углерода во всем пласте протяженностью $L=100$ м от давления, под которым закачивается жидкая двуокись углерода, представлена на рис. 7. Проницаемость пласта и температура инжектируемого диоксида углерода полагались равными $k_{0}=10^{-14} \mathrm{M}^{2}$ и $T_{e}=276$ К. Как следует из рисунка, при увеличении давления, под которым закачивается двуокись углерода, а также при уменьшении давления на правой границе пласта время замещения метана в газогидрате на диоксид углерода во всем пласте уменьшается. Это объясняется тем, что при увеличении перепада давления в пласте, в соответствии с законом Дарси, увеличивается скорость фильтрации двуокиси углерода, которая и определяет скорость замещения.

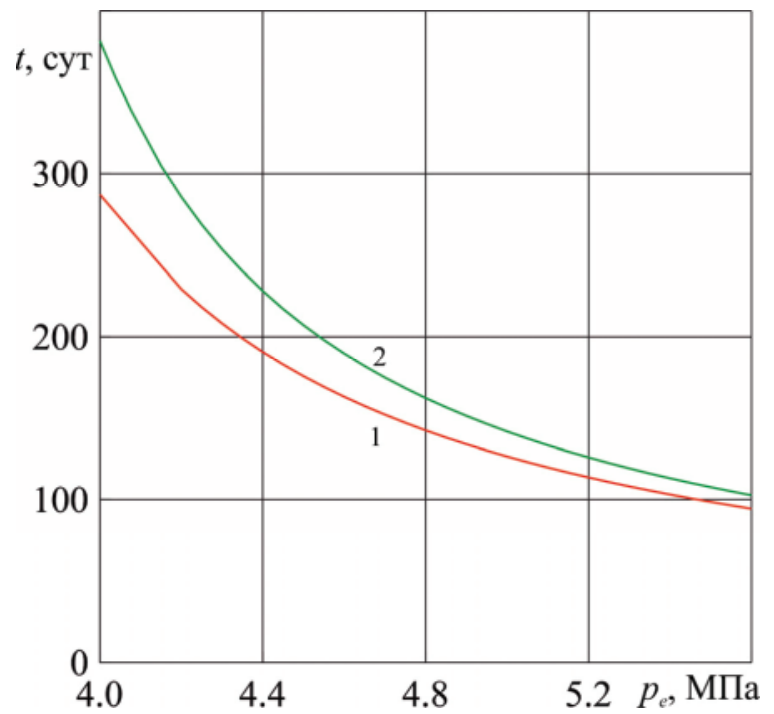

Pис. 7. Зависимость врелени полного замещения в газогидрате метана на диоксид углерода во всем пласте от давления закачиваемой двуокиси углерода. $p_{0}=3,2$ МПа (кривая 1) и 3,4 МПа (кривая 2)

Fig. 7. Time dependence of the total transition in methane hydrate to carbon dioxide hydrate in the entire formation on the pressure of the injected carbon dioxide. $p_{0}=3,2 \mathrm{MPa}$ (curve 1) and 3,4 MPa (curve 2) 


\section{Заключение}

Предложена математическая модель закачки жидкого диоксида углерода в газогидратный пласт конечной протяженности, сопровождающейся замещением метана на диоксид углерода в газогидрате. Результаты расчетов показали, что при низких значениях давления, под которым закачивается диоксид углерода, а также высоких значениях температуры инжектируемой двуокиси углерода, проницаемости и давления на правой границе пласта величи-

\section{СПИСОК ЛИТЕРАТУРЫ}

1. Methane exploitation by carbon dioxide from gas hydrates-phase equilibria for $\mathrm{CO}_{2}-\mathrm{CH}_{4}$ mixed hydrate system / K. Ohgaki, $\mathrm{K}$. Takano, H. Sangawa et al. // Journal of chemical engineering of Japan. - 1996. - V. 29. - P. 478-483.

2. Hirohama A., Shimoyama Yo., Wakabayashi A. Conversion of $\mathrm{CH}_{4}$-hydrate to $\mathrm{CO}_{2}$-hydrate in liquid $\mathrm{CO}_{2} / /$ Journal of chemical engineering of Japan. - 1996. - V. 29. - № 6. - P. 1014-1020.

3. Recovering methane from solid methane hydrate with carbon dioxide / H. Lee, S. Yongwon, Y.T. Sea et al. // Angewandte Chemie Int. Ed. - 2003. - V. 42. - P. 5048-5051

4. Lee B.R., Koh C.F., Sum A.K. Quantitative measurement and mechanisms for $\mathrm{CH}_{4}$ production from hydrates with the injection of liquid $\mathrm{CO}_{2}$ // Phys. Chem. Chem. Phys. - 2014. - V. 16. P. 14922-14927.

5. Replacement of $\mathrm{CH}_{4}$ in the hydrate by use of liquid $\mathrm{CO}_{2} / \mathrm{M}$. Ota, K. Morohashi, Yu. Abe et al. // Energy Conversion and Management. - 2005. - V. 46. - P. 1680-1691.

6. Methane recovery from methane hydrate using pressurized $\mathrm{CO}_{2} /$ M. Ota, Yu. Abe, M. Watanabe et al. // Fluid Phase Equilib. 2005. - V. 229. - P. 553-559.

7. Methane recovery from natural gas hydrate in porous sediment using pressurized liquid $\mathrm{CO}_{2} / \mathrm{Q}$. Yuan, C.Y. Sun, B. Liu et al. // Energy Convers. Management. - 2013. - V. 67. - P. 257-264.

8. Determination of appropriate condition on replacing methane from hydrate with carbon dioxide / X.T. Zhou, S.S. Fan, D.Q. Liang et al. // Energy Convers. Management. - 2008. - V. 49. P. 2124-2129.

9. Recovering methane from solid methane hydrate with carbon dioxide / H. Lee, Y. Seo, Y.T. Seo et al. // Angew Chem. Int. Ed. 2003. - V. 42. - P. 5048-5051.

10. Goel N. In situ methane hydrate dissociation with carbon dioxide sequestration: current knowledge and issues // J. Petrol Sci Eng. - 2006. - V. 51. - P. 169-184

11. Replacement of $\mathrm{CH}_{4}$ in hydrate in porous sediments with liquid $\mathrm{CO}_{2}$ injection / Y. Zhang, L.J. Xiong, X.S. Li et al. // Chem. Eng. Technol. - 2014. - V. 37. - № 12. - P. 2022-2029.

12. Донцов В.Е., Чернов А.А., Донцов Е.В. Ударные волны и образование гидрата углекислого газа при повышенном начальном давлении в газожидкостной среде // Теплофизика и аэромеханика. - 2007. - Т. 14. - № 1. - С. 23-39.

13. Донцов В.Е., Чернов А.А. Процессы растворения и гидратообразования за ударной волной в газожидкостной смеси // Доклады академии наук. - 2009. - Т. 425. - № 6. - С. 764-768.

14. Dontsov V.E., Chernov A.A. Dilution and hydrate forming process in shock waves // International Journal of Heat and Mass Transfer. - 2009. - V. 52. - № 21-22. - P. 4919-4928. на пластовой температуры в области, насыщенной метаном и его газогидратом, может подниматься выше равновесной температуры разложения газогидрата метана. Это соответствует возникновению второй границы фазовых переходов, на которой происходит разложение газогидрата метана на газ и воду, и появлению промежуточной области, насыщенной смесью метана и воды в свободном состоянии.

Исследование выполнено за счет гранта Российского научного фонда (проект № 17-79-20001).

15. Хасанов М.К., Шагапов В.Ш. Разложение газогидрата метана в пористой среде при инжекции теплого углекислого газа // Инженерно-физический журнал. - 2016. - № 5. C. $1129-1140$.

16. Шагапов В.Ш., Хасанов М.К. Режимы восстановления метана из газогидрата при инжекции «теплого» диоксида углерода в пористую среду // Теплофизика высоких температур. - 2017. T. 55. - № 5. - С. 753-761.

17. Хасанов М.К. Численное моделирование закачки углекислого газа в истощенное месторождение углеводородов // Известия Томского политехнического университета. Инжиниринг георесурсов. - 2017. - Т. 328. - № 8. - С. 48-54.

18. Бык С.Ш., Макогон Ю.Ф., Фомина В.И. Газовые гидраты. М.: Химия, 1980. -296 с.

19. Гималтдинов И.К., Хасанов М.К. Математическая модель образования газогидрата при инжекции газа в пласт, частично насыщенный льдом // Прикладная математика и механика. 2016. - T. 80. - № 1. - C. 80-90.

20. Шагапов В.Ш., Рафикова Г.Р., Хасанов М.К. К теории образования газогидрата в частично водонасыщенной пористой среде при нагнетании метана // Теплофизика высоких температур. 2016. - T. 54. - № 6. - C. 911-920.

21. Musakaev N.G., Borodin S.L. Numerical research of the gas hydrate dissociation to gas and ice in a reservoir during the gas extraction. // MATEC Web of Conferences. - 2017. - V. 115. № 05003. URL: https://www.matec-conferences.org/articles/matecconf/pdf/2017/29/matecconf_sts2017_05003.pdf (дата обращения 11.05.2018).

22. Шагапов В.Ш., Юмагулова Ю.А., Мусакаев Н.Г. Теоретическое исследование предельных режимов гидратообразования при контакте газа и воды // Прикладная механика и техническая физика. - 2017. - Т. 58. - № 2. - С. 3-15.

23. Чиглинцева А.С. Автомодельное решение задачи образования гидрата в снежном массиве // Вычислительная механика сплошных сред. - 2017. - Т. 10. - № 2. - С. 212-224.

24. Шагапов В.Ш., Чиглинцева А.С., Русинов А.А. Теоретическое моделирование процесса извлечения газа из пористого газогидратного пласта, частично насыщенного газом, с учетом теплового взаимодействия с окружающими породами // Теоретические основы химической технологии. - 2016. - Т. 50 . - № 4. C. $452-462$.

25. Истомин В.А., Якушев В.С. Газовые гидраты в природных условиях. - М.: Недра, 1992. - 236 с.

Поступила 14.06.2018 2. 


\section{Информация об авторах}

Хасанов M.K., кандидат физико-математических наук, доцент кафедры прикладной информатики и программирования Стерлитамакского филиала Башкирского государственного университета.

Столповский M.B., кандидат физико-математических наук, доцент кафедры физики Уфимского государственного нефтяного технического университета; научный сотрудник Стерлитамакского филиала Башкирского государственного университета.

Гималтдинов И.К., доктор физико-математических наук, профессор кафедры физики Уфимского государственного нефтяного технического университета. 


\title{
INJECTION OF LIQUID CARBON DIOXIDE IN RESERVOIR SATURATED BY METHANE AND ITS GAS HYDRATE
}

\author{
Marat K. Khasanov', \\ hasanovmk@mail.ru \\ Maxim V. Stolpovskii ${ }^{2}$, \\ s_maxim.pmm@mail.ru \\ Ilyas K. Gimaltdinov², \\ iljas_g@mail.ru \\ 1 Sterlitamak Branch of the Bashkir State University, \\ 49, Lenin Avenue, Sterlitamak, 453103, Russia. \\ 2 Ufa State Petroleum Technical University, \\ 1, Cosmonavtov street, Ufa, 450062, Russia
}

\begin{abstract}
The relevance of the study is related to development of theoretical foundations of gas production technologies from gas-hydrate deposits by substitution. The method of carbon dioxide injection is considered, which allows reducing significantly energy costs for development of natural gas hydrate deposits.

The main aim of the study is to determine the features of methane replacing with carbon dioxide in gas hydrate when injecting liquid carbon dioxide into gas hydrate formation.

Object: a porous layer of finite extent saturated with methane and its gas hydrate, the initial pressure and temperature of which correspond to the conditions of stable existence of methane gas hydrate.

Methods. On the basis of the equations of continuum mechanics, a mathematical model of heat and mass transfer in the natural reservoir is constructed, accompanied by methane substitution for carbon dioxide in gas hydrate. It is accepted that in the considered case in a layer there are two characteristic zones separated by a mobile boundary of phase transitions. The first (near) pore area is saturated with liquid carbon dioxide and its gas hydrate, and the second (far) area contains methane and its gas hydrate.

Result. The authors have obtained numerical solutions for pressure and temperature fields at injection of liquid carbon dioxide into a gas hydrate layer of finite extent and temperature dependences on the substitution border on injection pressure and formation permeability. It is established that at sufficiently low values of injection pressure and permeability the temperature on the boundary of substitution can rise above the equilibrium temperature of decomposition of methane gas hydrate into gas and water. This corresponds to the emergence of a second mobile interphase boundary at which decomposition of methane gas hydrate occurs. The authors determined the dependence of the maximum temperature of pumped liquid carbon dioxide, above which it is necessary to take into account the formation of a mixture of methane and water, on the pressure values at the right and left borders of the formation and its permeability. It is established that the mode with gas hydrate decomposition is implemented at high pressure values on the right boundary of the formation and low permeability and pressure values under which carbon dioxide is pumped. The authors obtained the dependences of the replacement boundary rate, as well as the time of complete replacement of methane gas hydrate by carbon dioxide gas hydrate in the entire formation, on the pressure on the right and left boundaries of the formation, as well as on its permeability.
\end{abstract}

Key words:

Methane hydrate, carbon dioxide, filtration, porous reservoir, replacement

This work was financially supported by the grant of the Russian Science Foundation (project 17-79-20001).

\section{REFERENCES}

1. Ohgaki K., Takano K., Sangawa H., Matsubara T., Nakano S. Methane exploitation by carbon dioxide from gas hydrates-phase equilibria for $\mathrm{CO}_{2}-\mathrm{CH}_{4}$ mixed hydrate system. $\mathrm{J}$ Chem Eng $\mathrm{Ja}$ pan, 1996, vol. 29, pp. 478-483.

2. Hirohama A., Shimoyama Yo., Wakabayashi A. Conversion of $\mathrm{CH}_{4}$-hydrate to $\mathrm{CO}_{2}$-hydrate in liquid $\mathrm{CO}_{2}$. Journal of chemical engineering of Japan, 1996, vol. 29, no. 6, pp. 1014-1020.

3. Lee H., Yongwon S., Sea Y.T. Recovering methane from solid methane hydrate with carbon dioxide. Angewandte Chemie Int. Ed, 2003, vol. 42, pp. 5048-5051.

4. Lee B.R., Koh C.F., Sum A.K. Quantitative measurement and mechanisms for $\mathrm{CH}_{4}$ production from hydrates with the injection of liquid $\mathrm{CO}_{2}$. Phys. Chem. Chem. Phys., 2014. vol. 16, pp. $14922-14927$.

5. Ota M., Morohashi K., Abe Yu. Replacement of $\mathrm{CH}_{4}$ in the hydrate by use of liquid $\mathrm{CO}_{2}$. Energy Conversion and Management, 2005, vol. 46, pp. 1680-1691.
6. Ota M., Abe Yu., Watanabe M. Methane recovery from methane hydrate using pressurized $\mathrm{CO}_{2}$. Fluid Phase Equilib., 2005, vol. 229, pp. 553-559.

7. Yuan Q., Sun C.Y., Liu B. Methane recovery from natural gas hydrate in porous sediment using pressurized liquid $\mathrm{CO}_{2}$. Energy Convers Management, 2013, vol. 67, pp. 257-264.

8. Zhou X.T., Fan S.S., Liang D.Q. Determination of appropriate condition on replacing methane from hydrate with carbon dioxide. Energy Convers Management, 2008, vol. 49, pp. 2124-2129.

9. Lee H., Seo Y., Seo Y.T. Recovering methane from solid methane hydrate with carbon dioxide. Angew Chem Int Ed., 2003, vol. 42, pp. 5048-5051.

10. Goel N. In situ methane hydrate dissociation with carbon dioxide sequestration: current knowledge and issues. J Petrol Sci Eng., 2006, vol. 51, pp. 169-184.

11. Zhang Y., Xiong L.J., Li X.S. Replacement of $\mathrm{CH}_{4}$ in hydrate in porous sediments with liquid $\mathrm{CO}_{2}$ injection. Chem Eng Technol., 2014, vol. 37 , no. 12 , pp. 2022-2029. 
12. Dontsov V.E., Chernov A.A., Dontsov E.V. Shock waves and formation of carbon dioxide hydrate at an increased pressure in the gas-liquid medium. Thermophysics and Aeromechanics, 2007, vol. 14, no. 1, pp. 21-35. In Rus.

13. Dontsov V.E., Chernov A.A. Dissolution and hydrate-formation processes behind the shock wave in a gas-liquid mixture. Doklady Physics, 2009, vol. 54, no. 4, pp. 215-219. In Rus.

14. Dontsov V.E., Chernov A.A. Dilution and hydrate forming process in shock waves. International Journal of Heat and Mass Transfer, 2009, vol. 52, pp. 4919-4928.

15. Khasanov M.K., Shagapov V.Sh. Methane gas hydrate decomposition in a porous medium upon injection of a warm carbon dioxide gas. Journal of Engineering Physics and Thermophysics, 2016, vol. 89, no. 5, pp. 1123-1133. In Rus.

16. Shagapov V.Sh., Khasanov M.K. Regimes of methane recovery from gas hydrate on injection of «warm» carbon dioxide into a porous medium. High Temperature, 2017, vol. 55, no. 5, pp. 737-745. In Rus.

17. Khasanov M.K. Numerical simulation of carbon dioxide injection into a depleted hydrocarbon deposit. Bulletin of the Tomsk Polytechnic University. Geo Assets Engineering, 2017, vol. 328, no. 8, pp. 48-54. In Rus.

18. Byk S.Sh., Makogon Yu.F., Fomina V.I. Gazovye gidraty [Gas hydrate]. Moscow, Khimiya Publ., 1980. 296 p.

19. Gimaltdinov I.K., Khasanov M.K. Mathematical model of the formation of a gas hydrate on the injection of gas into a stratum partially saturated with ice, Journal of Applied Mathematics and Mechanics, 2016, vol. 80, no. 1, pp. 57-64. In Rus.
20. Shagapov V.S., Rafikova G.R., Khasanov M.K. On the theory of formation of gas hydrate in partially water-saturated porous medium when injecting methane. High Temperature, 2016, vol. 54, no. 6, pp. 858-866. In Rus.

21. Musakaev N.G., Borodin S.L. Numerical research of the gas hydrate dissociation to gas and ice in a reservoir during the gas extraction. MATEC Web of Conferences, 2017, vol. 115, no. 05003. Available at: https://www.matec-conferences.org/articles/matecconf/pdf/2017/29/matecconf_sts2017_05003.pdf (accessed 11 May 2018).

22. Shagapov V.Sh., Yumagulova Yu.A., Musakaev N.G. Theoretical study of the limiting regimes of hydrate formation during contact of gas and water. J. of Applied Mechanics and Technical Physics, 2017, vol. 58, no. 2, pp. 189-199. In Rus.

23. Chiglintseva A.S. Self-similar solution of the problem of hydrate formation in snow massifs. Computational Continuum Mechanics, 2017, vol. 10, no. 2, pp. 212-224. In Rus.

24. Shagapov V.Sh., Chiglintseva A.S., Rusinov A.A. Theoretical modeling of gas extraction from a partially gas-saturated porous gas-hydrate reservoir with respect to thermal interactions with surrounding rocks. Theoretical Foundations of Chemical Engineering, 2016, vol. 50, no. 4, pp. 449-458. In Rus.

25. Istomin V.A., Yakushev V.S. Gazovye gidraty v prirodnykh usloviyakh [Gas Hydrates under Natural Conditions]. Moscow, Nedra Publ., 1992. 236 p.

Received: 14 June 2018.

\section{Information about the authors}

Marat K. Khasanov, Cand. Sc., associate professor, Sterlitamak Branch of the Bashkir State University

Maxim V. Stolpovskii, Cand. Sc., associate professor, Ufa State Petroleum Technical University; researcher, Sterlitamak Branch of the Bashkir State University

Ilyas K. Gimaltdinov, Dr. Sc., professor, Ufa State Petroleum Technical University. 